\title{
Double improved integral adaptive control of one order system
}

$$
\text { Yuliang Chen }{ }^{1, a} \text {, Lingling Wang }{ }^{2, b} \text {, Xiaoyan } \mathrm{Qu}^{1, \mathrm{c}}
$$

${ }^{1}$ Department of Ordnance Science and Technology, Naval aeronautical and astronautical University Yantai, China

${ }^{2}$ Department of control engineering, Naval Aeronautical and Astronautical University, Yantai, China achengyuliang1024@126.com, 'quxiaoyan1024@126.com

Keywords: adaptive control, parameter identification, one order system

\begin{abstract}
In order to achieve adaptive control and single parameter identification of one order system, an ordinary adaptive control method is used in this paper. By introducing integral control and considering multiple variables, an adaptive control law is designed, and the result of parameter identification is analyzed. In the end, a conclusion can be made that theoretical analysis is correct and parameter identification method is effective by numerical simulation.
\end{abstract}

\section{Introduction}

Integral control is an classic control strategy and it is use in most control system to reduce system error ${ }^{[1-7]}$. As the demand for control performance increases, the problem of time-variance parameters of controlled system is more and more important. A lot of parameter identification method is proposed by specialists and representatives from China and abroad. The classical parameter identification method is least square method ${ }^{[1]}$, Kalman filtering method, adaptive. The method modern parameter identification method is neural network method ${ }^{[2]}$, genetic algorithm method $^{[3]}$ and particle swarm optimization. But calculating amount of these method is large, instantaneity and astringency can't meet the demands of the control system. In this paper, a double improved integral adaptive control ${ }^{[8-11]}$ is proposed, the simulation results show that system parameters can be identified.

\section{Problem Description}

One order system can be written as:

$$
\dot{x}=a x+u
$$

where $a$ is unknown constant parameter, the goal is designing a controller such that the system state $x$ can trace the expected value $x^{d}$.

\section{Design Double Improved Integral Adaptive Identification Controller}

An ordinary adaptive control method is used as follows, define a error variable as $z_{1}=x_{1}-x_{1}^{d}$, then

$$
\dot{z}_{1}=\dot{x}_{1}-\dot{x}_{1}^{d}=a x+u
$$

Design state feedback control law as:

$$
u=-\hat{a} x-\sum_{i=1}^{n} k_{i} f_{i}\left(z_{1}\right)-k_{s 1} \int z_{1} d t
$$

Choose $n=1, k_{i}>0, f_{1}\left(z_{1}\right)=z_{1}$. design regulating law:

$$
\dot{\hat{a}}=\Gamma_{a} z_{1} x
$$

Define $z_{2}=\int z_{1} d t, \quad \tilde{a}=a-\hat{a}$, then: 


$$
\begin{aligned}
& \dot{z}_{1}=\tilde{a} x-k_{s_{1}} z_{2}-k_{1} z_{1} \\
& \dot{z}_{2}=z_{1} \\
& \dot{\tilde{a}}=-\Gamma_{a} z_{1} x
\end{aligned}
$$

choose Lyapunov function:

$$
V=\frac{1}{2} z_{1}^{2}+\frac{k_{s 1}}{2} z_{2}^{2}+\frac{1}{2 \Gamma_{a}} \tilde{a}^{2}
$$

Then:

$$
\dot{V}=\tilde{a} x z_{1}-k_{1} z_{1}^{2}-k_{s_{1}} z_{2} z_{1}+k_{s 1} z_{2} z_{1}+\frac{1}{\Gamma_{a}} \tilde{a} \dot{\tilde{a}}=-k_{1} z_{1}^{2} \leq 0
$$

The system is stable, and there is $z_{1} \rightarrow 0$, but it can't make sure that system parameters can be identified.

The above model can be written as:

$$
\left[\begin{array}{c}
\dot{z}_{1} \\
\dot{z}_{2} \\
\dot{\tilde{\alpha}}
\end{array}\right]=\left[\begin{array}{ccc}
-k_{1} & -k_{s_{1}} & x \\
1 & 0 & 0 \\
-\Gamma_{a} x & 0 & 0
\end{array}\right]\left[\begin{array}{c}
z_{1} \\
z_{2} \\
\tilde{\alpha}
\end{array}\right]
$$

Obviously, system can make sure all state identified. Define:

$$
\dot{z}_{2}=z_{1}-k_{2} z_{2}
$$

Then:

$$
\left[\begin{array}{c}
\dot{z}_{1} \\
\dot{z}_{2} \\
\dot{\tilde{\alpha}}
\end{array}\right]=\left[\begin{array}{ccc}
-k_{1} & -k_{s_{1}} & x \\
1 & -k_{2} & 0 \\
-\Gamma_{a} x & 0 & 0
\end{array}\right]\left[\begin{array}{c}
z_{1} \\
z_{2} \\
\tilde{\alpha}
\end{array}\right]
$$

There is $z_{1} \rightarrow 0, z_{2} \rightarrow 0$, then

$$
\dot{z}_{1}=\tilde{a} x-k_{s_{1}} z_{2}-k_{1} z_{1}=\tilde{a} x=0
$$

When $x \neq 0$, unknown system parameters can be identified. That is $\tilde{a}=0$.

Obviously, the improved integral adaptive identification controller can ensure the system parameters be identified. Considering the third variable, then design:

$$
\dot{\hat{a}}=\Gamma_{a} z_{1} x-k_{a d 1} \hat{a}
$$

Then:

$$
\dot{\tilde{a}}=-\dot{\hat{a}}=-\Gamma_{a} Z_{1} X+k_{a d 1} \hat{a}=-\Gamma_{a} Z_{1} x+k_{a d 1}(a-\tilde{a})
$$

Then:

$$
\left[\begin{array}{c}
\dot{z}_{1} \\
\dot{z}_{2} \\
\dot{\tilde{\alpha}}
\end{array}\right]=\left[\begin{array}{ccc}
-k_{1} & -k_{s_{1}} & x \\
1 & -k_{2} & 0 \\
-\Gamma_{a} x & 0 & -k_{a d 1}
\end{array}\right]\left[\begin{array}{c}
z_{1} \\
z_{2} \\
\tilde{\alpha}
\end{array}\right]+\left[\begin{array}{c}
0 \\
0 \\
k_{a d 1} a
\end{array}\right]
$$

The balance point of system is:

$$
\left[\begin{array}{c}
z_{1} \\
z_{2} \\
\tilde{\alpha}
\end{array}\right]=\left[\begin{array}{ccc}
-k_{1} & -k_{s_{1}} & x \\
1 & -k_{2} & 0 \\
-\Gamma_{a} x & 0 & -k_{a d 1}
\end{array}\right]^{-1}\left[\begin{array}{c}
0 \\
0 \\
k_{a d 1} a
\end{array}\right] \neq\left[\begin{array}{l}
0 \\
0 \\
0
\end{array}\right]
$$

It can't ensure system converge to zero.

Define that $a$ is the forth state of system, then: 


$$
\left[\begin{array}{c}
\dot{z}_{1} \\
\dot{z}_{2} \\
\dot{\tilde{\alpha}} \\
\dot{a}
\end{array}\right]=\left[\begin{array}{cccc}
-k_{1} & -k_{s_{1}} & x & 0 \\
1 & -k_{2} & 0 & 0 \\
-\Gamma_{a} x & 0 & -k_{a d 1} & k_{a d 1} \\
0 & 0 & 0 & 0
\end{array}\right]\left[\begin{array}{c}
z_{1} \\
z_{2} \\
\tilde{\alpha} \\
a
\end{array}\right]
$$

It can’t ensure all state of system converge to zero.

\section{Numerical Simulation}

Choose unknown parameter $a=3$, initial state $x_{1}(0)=-1$, expected state $x_{1}^{d}=1$, use Simulink in Matlab, write program with double improved integral adaptive identification controller, the program can be written as:

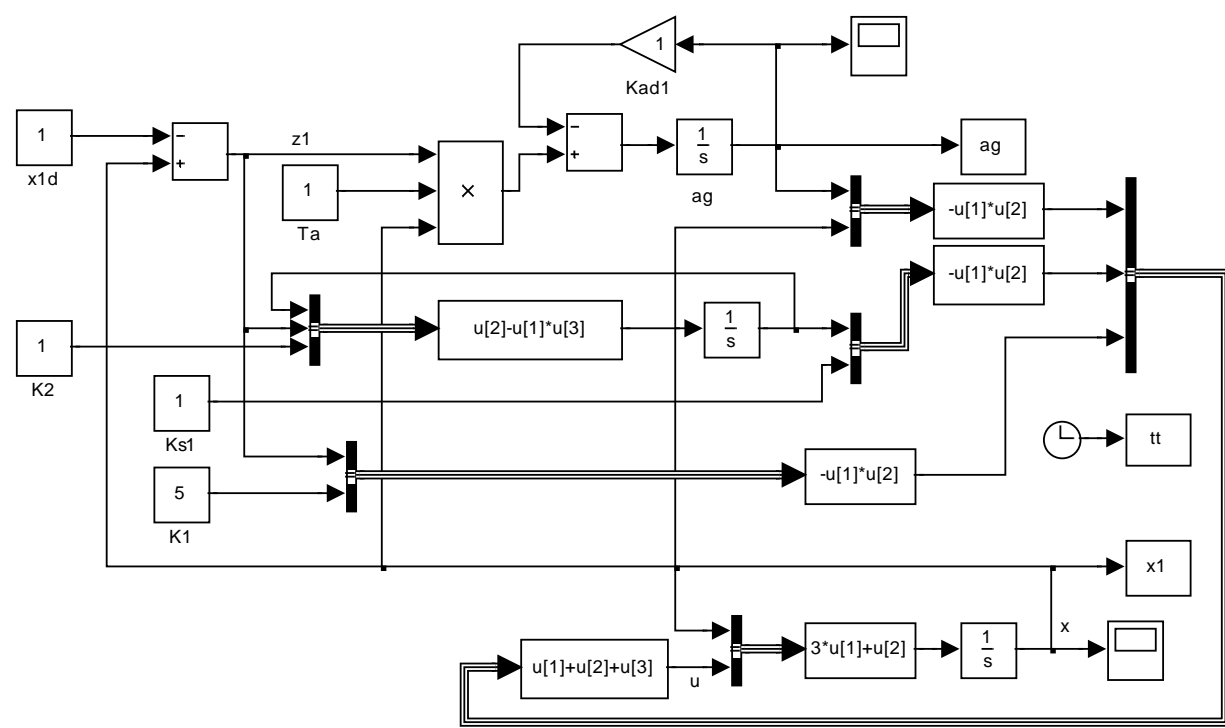

Fig.1 Program

Choose $k_{1}=5, k_{s 1}=1, \Gamma_{a}=1, k_{2}=1, k_{a d 1}=1$, the simulation results are as follows:

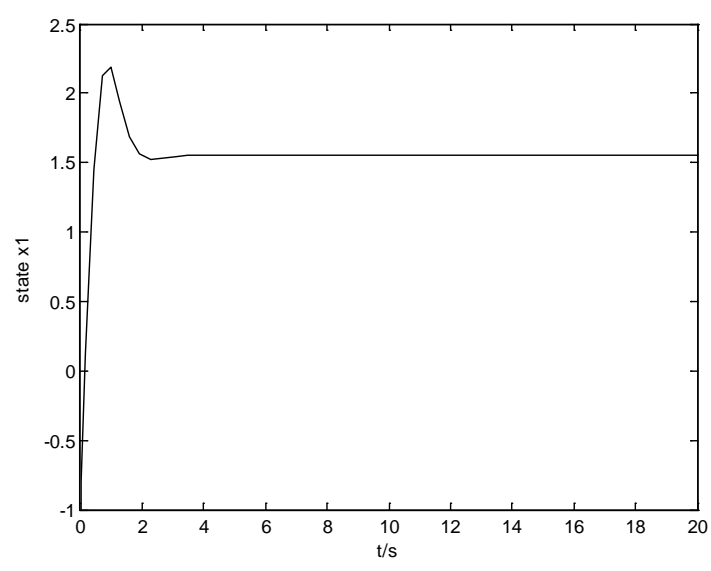

Fig.2 state a

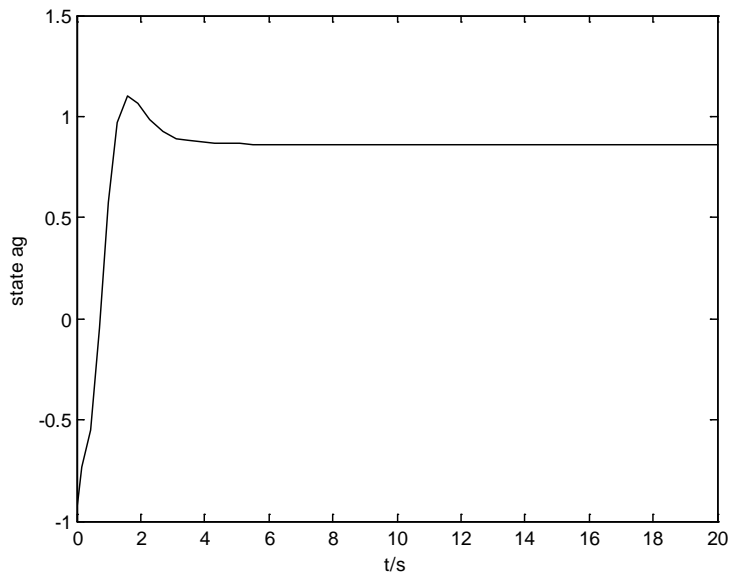

Fig.3 state $\mathrm{x} 1$

From the result, the steady state error and state estimate error of system both can't converge to zero, choose $k_{1}=50, k_{s 1}=1, \Gamma_{a}=1, k_{2}=1, k_{a d 1}=1$, the simulation results are as follows: 


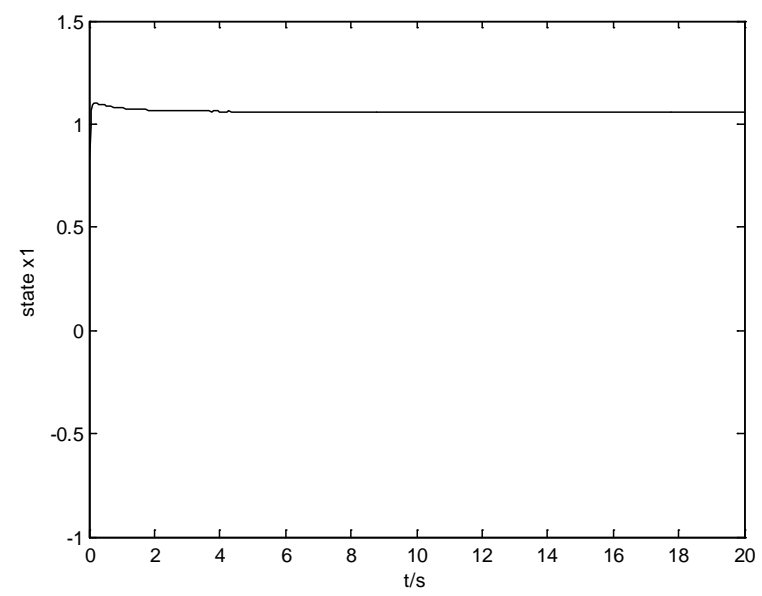

Fig.4 state a

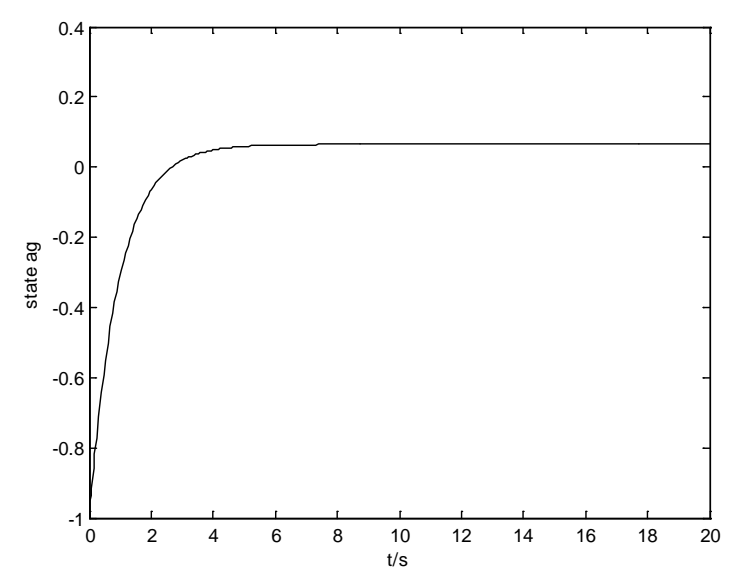

Fig. 5 state $\mathrm{x} 1$

Choose $k_{1}=50, k_{s 1}=0, \Gamma_{a}=1, k_{2}=1, k_{a d 1}=0$, the simulation results are as follows:

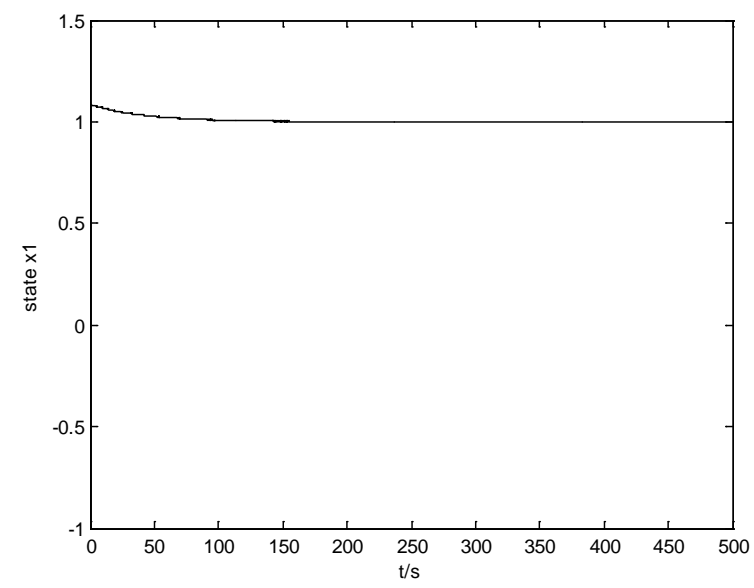

Fig.6 state a

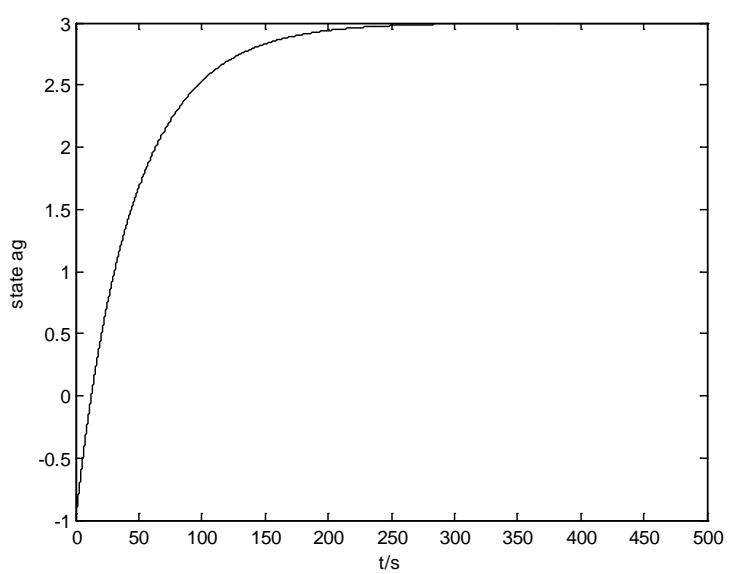

Fig.7 state $\mathrm{x} 1$

Choose $k_{1}=5, k_{s 1}=0, \Gamma_{a}=1, k_{2}=1, k_{a d 1}=0$, the simulation results are as follows:

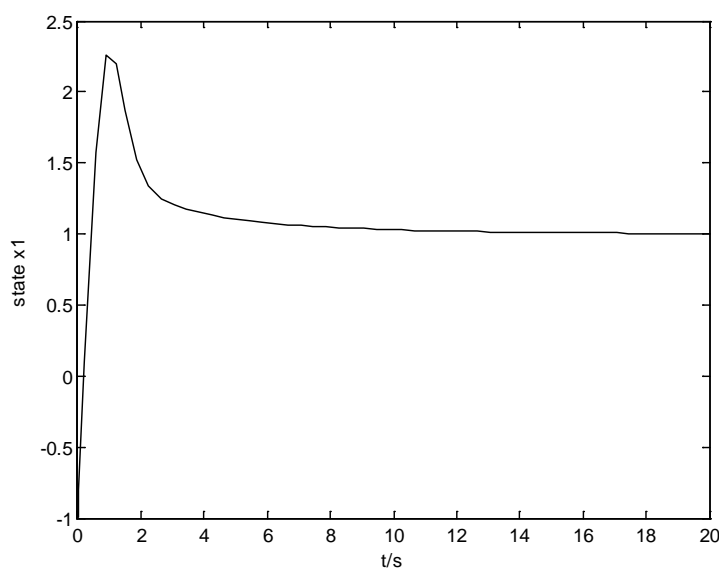

Fig.8 state a

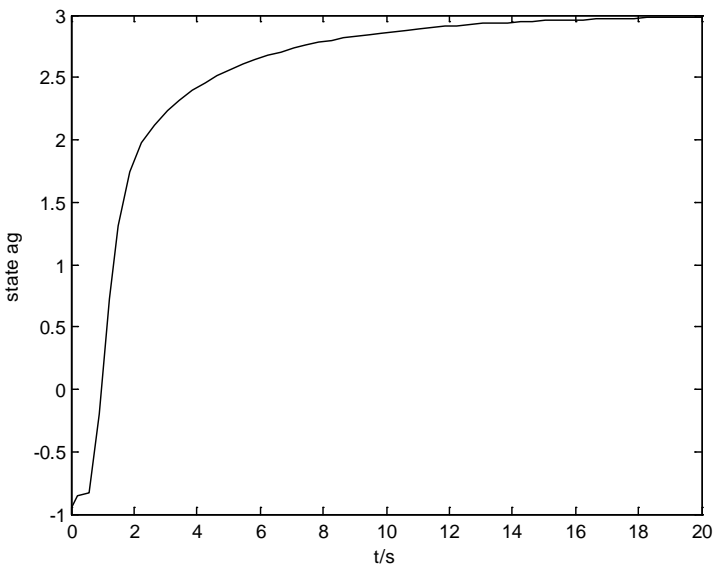

Fig.9 state $\mathrm{x} 1$

The convergence time is 20s. The result shows that if control error converge quickly, it will go against parameter identification. If control gain is small, the unknown parameter can be identified quickly. 


\section{Conclusion}

According to the problem that system parameter need to be identified, a double improved integral adaptive control is proposed in this paper. By analysing theory and the simulation results, we can make a conclusion that the improved integral adaptive control method is effective to parameter identification. But if the control gain is too large, it will go against parameter identification.

\section{Reference}

[1] Maurizio C, Marcello P, Giansalvo, et al. A new experimental application of least-squares techniques for the estimation of the induction motor parameters[J]. IEEE Transactions on Industry Applications, 2003, 39(5):1247-1256.

[2] Raj M B, Alexander G P, Hamid A T, Neural speed filtering for sensorless induction motor drives[J]. Control Engineering Practice, 2004, 12(6): 687-706.

[3] Huang K S, Kent W, Wu Q, et al. Parameter identification of an induction machine using genetic algorithms[C] // Proceedings of the 1999 IEEE International Symposium on Computer Aided Control System Design. [S. L.]: [s. N.],1999: 510-515.

[4] Huynh D C, Dunnugan M W. Parameter estimation of an induction machine using advanced particle swarm optimisation algorithms[J]. IET Electric Power Applications, 2010, 4(9): 748-760.

[5] David K. Schmidt,James Stevens,Jason Roney.Dynamic Modeling,Control, and Station-Keeping Guidance of A Large High-Altitude“Near-Space” Airship.AIAA Guidance,Navigation,and Control Conference and Exhibit. 2006-6781

[6] David K. Schmidt.Modeling and Near-Space Station-Keeping Control of a Large High-Altitude Airship.Journal of Control and Dynamics,2007,30(2):540 547

[7] David K. Schmidt,James Stevens,Jason Roney.Near-Space Station-Keeping Performance of a Large High-Altitude Notional Airship.Journal of Control and Dynamics,2007,44(2):611 615

[8] Donald J.McTavish,Kyle Davidson.Practical Large-Motion Modeling of Geometrically Complex Flexible Vehicles:A Consistent-Mass Standard-FEM Based,All Terms Included Formulation.47th AIAA/ASME/ASCE/AHS/ASC Structures,Structural Dynamics,and Materials Conference.2006-1664

[9] Duc Cuong Quach, Shuang Huang, Quan Yin, Chunjie Zhou. An improved Direct Adaptive Fuzzy controller for an uncertain DC Motor Speed Control System. TElkomnika, indonesian journal of electrical engineering. February 2013; Vol 11, No 2: 1083-1092.

[10] Yonghong Zhu, Qing Feng, Jianhong Wang. Neural Network-based Adaptive Passive Output Feedback Control for MIMO Uncertain System. TElkomnika, indonesian journal of electrical engineering. October 2012; Vol 10, No 6: 1263-1272.

[11] Wen Xinling, Chen Yu. Research of the Nonlinear System Identification Based on the Volterra RLS Adaptive Filter Algorithm. TElkomnika, indonesian journal of electrical engineering. May 2013; Vol 11, No 5: 2277-2283. 